\title{
GEODESICS AND CONFORMAL TRANSFORMATIONS OF HEISENBERG-REITER SPACES
}

\author{
J. F. TORRES LOPERA
}

\begin{abstract}
Generalized Heisenberg groups, in the sense of Reiter, can be endowed with left-invariant metrics whose geodesics and curvature are obtained. Using these curvature data it is also proved that on their nilmanifolds (compact or not), every conformal transformation is in fact an isometry. A large family of nonisometric examples is given.
\end{abstract}

\section{Introduction and main results.}

DEFINITION. A generalized Heisenberg group in the sense of Reiter [10] is the product $G_{B}=X \times Y \times Z$ of three Abelian topological groups $X, Y, Z$, endowed with the law

$$
(x, y, z)\left(x^{\prime}, y^{\prime}, z^{\prime}\right)=\left(x+x^{\prime}, y+y^{\prime}, z+z^{\prime}+B\left(x, y^{\prime}\right)\right),
$$

where $B: X \times Y \rightarrow Z$ is a nonzero, continuous and $\mathbf{Z}$-bilinear map, ( $\mathbf{Z}$ the ring of integer numbers).

This family of two-step nilpotent groups includes as particular cases Haraguchi's generalized Heisenberg groups [1], their complex, quaternionic and Cayley analogues, Kaplan's groups of Heisenberg type [3], and many others [7]. Let us call $\pi_{X}: \tilde{X} \rightarrow X$ the universal covering of a topological space $X$. It is easy to prove that, under natural hypothesis, the universal covering group $\tilde{G}_{B}=\tilde{X} \times \tilde{Y} \times \tilde{Z}$ of a generalized "Heisenberg-Reiter group" (HR-group for short), is HR too, with group law associated to $\tilde{B}: \tilde{Y} \times \tilde{Y} \rightarrow \tilde{Z}$, the unique lifting of $\left(\pi_{X} \times \pi_{Y}\right) B$ sending $(0,0)$ into $0[\mathbf{7}]$. In the Lie-group case, $\tilde{X}, \tilde{Y}, \tilde{Z}$ are finite dimensional real vector spaces, and $\tilde{B}$ (in the sequel denoted $B$ for short), is in fact $\mathbf{R}$-linear ( $\mathbf{R}$ the field of real numbers). From these facts and a theorem of $\mathrm{Mal}^{\prime} \mathrm{cev},[\mathbf{8}]$, it follows that every homogeneous space of a Lie HR-group $G_{B}$ can be obtained from its universal covering $\tilde{G}_{B}$ via the natural action of a discrete subgroup $\Gamma$ of $G_{B}$. Therefore, we will deal mainly with the case of $G_{B}$ being such a product of three real vector space $X, Y, Z$, all other HR-nilmanifolds being obtained in the preceding way when necessary. On $G_{B}$ we consider the global coframe of left-invariant 1-forms

$$
\xi^{i}=d x^{i}, \quad \eta^{u}=d y^{u}, \quad \varsigma^{\alpha}=d z^{\alpha}-\sum_{i, u} B_{i u}^{\alpha} x^{i} d y^{u}
$$

Received by the editors May 30, 1986.

1980 Mathematics Subject (lassification (1985 Revision). Primary 53A20, 53C22, 53C25, 53C30.

Key words and phrases. Generalized Heisenberg group, nilmanifold, geodesic, conformal transformation, Weyl conformal tensor. 
where $x^{i}, y^{u}, z^{\alpha}$ are the coordinate functions induced on $G_{B}$ by bases of $X, Y, Z$, $\left\{e_{i} ; i \in \Xi\right\},\left\{f_{u} ; u \in \Upsilon\right\},\left\{g_{\alpha} ; \alpha \in \Theta\right\}$, respectively, and where

$$
B\left(e_{i}, f_{u}\right)=\sum_{\alpha} B_{i u}^{\alpha} g_{\alpha}
$$

Further mention to these finite sets $\Xi, \Upsilon, \Theta$, where indices $i, u, \alpha$ range, will be avoided as much as possible. Let us fix the following left-invariant metric tensor $g$ on the HR-group $G_{B}$ :

$$
g=\sum_{i}\left(\xi^{i}\right)^{2}+\sum_{u}\left(\eta^{u}\right)^{2}+\sum_{\alpha}\left(\varsigma^{\alpha}\right)^{2}
$$

The moving frame of left-invariant vector fields dual to (1) is

$$
X_{i}=\frac{\partial}{\partial x^{i}}, \quad Y_{u}=\frac{\partial}{\partial y^{u}}+\sum_{i, \alpha} B_{i u}^{\alpha} x^{i} \frac{\partial}{\partial z^{\alpha}}, \quad Z_{\alpha}=\frac{\partial}{\partial z^{\alpha}},
$$

and it is orthonormal with respect to $g,(3)$.

The main results in this paper are Theorems 1 and 2, describing the geodesic lines of $\left(G_{B}, g\right)$ and the conformal transformations of their nilmanifolds, respectively.

THEOREM 1. The geodesic line of $\left(G_{B}, g\right)$ with initial conditions $\left(x_{0}, y_{0}, z_{0}\right)$, $\left(\dot{x}_{0}, \dot{y}_{0}, \dot{z}_{0}\right)$ is given by the analytic vector valued maps of a real variable:

(5) $x(t)=[(1 / F)(\mathrm{id}-\cos (t \sqrt{F}))] M+[(1 / \sqrt{F}) \sin (t \sqrt{F})] \dot{\xi}_{0}+[\cos (t \sqrt{F})] x_{0}$,

(6) $y(t)=[(1 / H)(\mathrm{id}-\cos (t \sqrt{H}))] N+[(1 / \sqrt{H}) \sin (t \sqrt{H})] \dot{\eta}_{0}+[\cos (t \sqrt{H})] y_{0}$,

(7) $z(t)=z_{0}+t \sum_{\alpha} E^{\alpha} g_{\alpha}+\sum_{\alpha}\left(\sum_{i, j} Q_{i j}^{\alpha} \int_{0}^{t} x^{i}(s) x^{j}(s) d s\right) g_{\alpha}$

$$
\begin{aligned}
+\sum_{i, u, \alpha} B_{i u}^{\alpha} D^{u}\left(\left[\frac{1}{\sqrt{F}} \sin (t \sqrt{F})\right] x_{0}+\left[\frac{1}{F}\left(t \mathrm{id}-\frac{1}{\sqrt{F}} \sin (t \sqrt{F})\right)\right] M\right. \\
\left.+\left[\frac{1}{F}(\mathrm{id}-\cos (t \sqrt{F}))\right] \dot{\xi}_{0}\right)^{i} g_{\alpha}
\end{aligned}
$$

where

$$
\begin{gathered}
\dot{\xi}_{0}=\sum_{i} \dot{x}_{0}^{i} e_{i}, \quad \dot{\eta}_{0}=\sum_{u} \dot{y}_{0}^{u} f_{u}, \quad \dot{x}_{0}=\sum_{i} \dot{x}_{0}^{i}\left(\partial / \partial x^{i}\right), \\
\dot{y}_{0}=\sum_{u} \dot{y}_{0}^{u}\left(\partial / \partial y^{u}\right), \quad \dot{z}_{0}=\sum_{\alpha} \dot{z}_{0}^{\alpha}\left(\partial / \partial z^{\alpha}\right) \\
x(t)=\sum_{i} x(t)^{i} e_{i}, \quad y(t)=\sum_{u} y(t)^{u} f_{u}, \quad z(t)=\sum_{\alpha} z(t)^{\alpha} g_{\alpha}, \\
E^{\alpha}=\dot{z}_{0}^{\alpha}-\sum_{i, u} B_{i u}^{\alpha} x_{0}^{i} \dot{y}_{0}^{u}, \quad D^{u}=\dot{y}_{0}^{u}-\sum_{i, \alpha} B_{i u}^{\alpha} x_{0}^{i} E^{\alpha}, \\
C^{i}=\dot{x}_{0}^{i}+\sum_{u, \alpha} B_{i u}^{\alpha} y_{0}^{u} E^{\alpha}, \quad F_{i j}=\sum_{\alpha, \beta, u} B_{i u}^{\alpha} B_{j u}^{\beta} E^{\alpha} E^{\beta}, \\
M^{i}=-\sum_{u, \alpha} B_{i u}^{\alpha} D^{u} E^{\alpha}, \quad H_{u v}=\sum_{\alpha, \beta, i} B_{i u}^{\alpha} B_{i v}^{\beta} E^{\alpha} E^{\beta}, \\
N^{u}=\sum_{i, \alpha} B_{i u}^{\alpha} C^{i} E^{\alpha}, \quad Q_{i j}^{\alpha}=\sum_{u, \beta} B_{i u}^{\alpha} B_{j u}^{\beta} E^{\beta}
\end{gathered}
$$


$F$ and $H$ being matrices with components $F_{i j}, H_{u v}$ respectively, and where the functions enclosed between brackets are matrix-valued analytic in the real variable $t$, and obtained after substitution in the corresponding complex variable power series the scalar variable by $t F$ or $t H$ in each case.

THEOREM 2. Let $\Gamma$ be an arbitrary discrete subgroup of a HR-Lie group $G_{B}$ and $\bar{g}$ the Riemannian metric induced on $M=\Gamma \backslash G_{B}$ by the metric $g$, (1), on the universal covering group $\tilde{G}_{B}$ of $G_{B}$. Then we have:

(1) $(M, \bar{g})$ is not projectively flat,

(2) $(M, \bar{g})$ is not conformally flat,

(3) Every conformal transformation on $(M, \bar{g})$ must be an isometry.

Moreover, these Riemannian HR-nilmanifolds $(M, \bar{g})$ are not Einstein, and under suitable hypothesis we infer that they are not locally symmetric, nor $\mathfrak{S}$-spaces in the sense of Sasaki-Yano either. The first integer and real cohomology group of compact HR-nilmanifolds have been studied in [7] . Here the volume of compact quotients is computed for a large family of HR-nilmanifolds, yielding nonisometric examples.

2. Proof of Theorem 1. The Lagrangian function $L$ associated to the metric (3) of $\S 1$ is defined by

$$
L(v)=\sum_{i}\left(\dot{x}^{i}\right)^{2}+\sum_{u}\left(\dot{y}^{u}\right)^{2}+\sum_{\alpha}\left(\dot{z}^{\alpha}-\sum_{i u} B_{i u}^{\alpha} x^{i} \dot{y}^{u}\right)^{2}
$$

where

$$
v=\sum_{i} \dot{x}^{i}\left(\frac{\partial}{\partial x^{i}}\right)+\sum_{u} \dot{y}^{u}\left(\frac{\partial}{\partial y^{u}}\right)+\sum_{\alpha} \dot{z}^{\alpha}\left(\frac{\partial}{\partial z^{\alpha}}\right)
$$

is an arbitrary vector tangent to $G_{B}$. The geodesics of $\left(G_{B}, g\right)$ are the solutions of the Euler-Lagrange equations:

$$
\begin{gathered}
(d / d t)\left(\partial L / \partial \dot{x}^{i}\right)-\left(\partial L / \partial x^{i}\right)=0 \\
(d / d t)\left(\partial L / \partial \dot{y}^{u}\right)-\left(\partial L / \partial y^{u}\right)=0 \\
(d / d t)\left(\partial L / \partial \dot{z}^{\alpha}\right)-\left(\partial L / \partial z^{\alpha}\right)=0 .
\end{gathered}
$$

From $(1)$ and $(2 \alpha)$ it follows that on every geodesic:

$$
\dot{z}^{\alpha}-\sum_{i, u} B_{i u}^{\alpha} x^{i} \dot{y}^{u}=E^{\alpha} \quad \text { (constant). }
$$

Using (4) in (2i) and (2u) one obtains

$$
\begin{aligned}
\dot{y}^{u}-\sum_{i, \alpha} B_{i u}^{\alpha} x^{i} E^{\alpha} & =D^{u} \quad \text { (constant) }, \\
\dot{x}^{i}+\sum_{u, \alpha} B_{i u}^{\alpha} y^{u} E^{\alpha} & =C^{i} \quad \text { (constant), }
\end{aligned}
$$

on each geodesic. Differentiating with respect to $t$ in (5) and (6) one has

$$
\ddot{x}^{i}=-\sum_{j} F_{i j} x^{j}+M^{i}
$$


where $F=\left(F_{i j}\right)$ and $M=\left(M^{i}\right)$ are defined above. Calling

$$
a^{i}(t)=\dot{x}^{i}, \quad b^{i}(t)=x^{i}, \quad W=\left(\begin{array}{cc}
0 & -F \\
\operatorname{id} & 0
\end{array}\right)
$$

with id the identity operator, equations (7) can be rewritten as a linear differential system with constant coefficients:

$$
\left(\frac{d}{d t}\right)\left(\begin{array}{l}
a \\
b
\end{array}\right)=\left(\begin{array}{cc}
0 & -F \\
\mathrm{id} & 0
\end{array}\right)\left(\begin{array}{l}
a \\
b
\end{array}\right)+\left(\begin{array}{c}
M \\
0
\end{array}\right) .
$$

The general solution of $(8)$ is

$$
\left(\begin{array}{c}
a(t) \\
b(t)
\end{array}\right)=\exp (t W)\left(\int_{0}^{t} \exp (-s W)\left(\begin{array}{c}
M \\
0
\end{array}\right) d s+\left(\begin{array}{c}
\dot{x}_{0} \\
x_{0}
\end{array}\right)\right)
$$

and since

$$
\exp (t W)=\left(\begin{array}{cc}
\cos (t \sqrt{F}) & (-\sqrt{F}) \sin (t \sqrt{F}) \\
(1 / \sqrt{F}) \sin (t \sqrt{F}) & \cos (t \sqrt{F})
\end{array}\right)
$$

we obtain that $\S 1(5)$ is the $X$-component $x(t)$ of the geodesic. Abusing notation, we have written $\dot{x}_{0}$ instead of $\dot{\xi}_{0}$. After a quite analogous computation one gets formula $\S 1(6)$ for $y(t)$. To reach $z(t)$, we integrate (4) using (5) and $\S 1(5)$.

$$
\text { (11) } \begin{aligned}
z(t) & =z_{0}+\sum_{\alpha}\left(\int_{0}^{t} z^{\alpha}(s) d s\right) g_{\alpha} \\
& =z_{0}+\sum_{\alpha}\left(t E^{\alpha}+\sum_{i, u} B_{i u}^{\alpha} D^{u} \int_{0}^{t} x(s)^{i} d s+\sum_{i, j} Q_{i j}^{\alpha} \int_{0}^{t} x(s)^{i} x(s)^{j} d s\right) g_{\alpha} .
\end{aligned}
$$

The first integral is determined after a term by term integration of the corresponding matric series in $x(t), \S 1(5)$, picking then the $i$ th component

$$
\begin{aligned}
\int_{0}^{t} x(s) d s=\sum_{i}\left([(1 / \sqrt{F}) \sin (t \sqrt{F})] x_{0}+[(1 / F)(\right. & t \mathrm{id}-(1 / \sqrt{F}) \sin (t \sqrt{F}))] M \\
& \left.+[(1 / F)(\mathrm{id}-\cos (t \sqrt{F}))] \dot{\xi}_{0}\right)^{i} e_{i}
\end{aligned}
$$

hence $\S 1(7)$ follows.

The integral $\sum_{i, j} Q_{i j}^{\alpha} \int_{0}^{t} x^{i}(s) x^{j}(s) d s$ can be expressed in terms of "elementary functions", as those above, in some remarkable cases. Let us denote as $M_{r s}$ the additive group of real matrices with $r$ rows and $s$ columns. Set $X=M_{p q}, Y=M_{q r}$, $Z=M_{p r}$. The usual matrix product $B: X \times Y \rightarrow Z$ induces a group law on the product $X \times Y \times Z$ as in $\S 1(1)$ which makes it a HR-group, denoted $H_{\mathbf{R}}(p, q, r)$. For $p=1$, we have $Q_{i j}^{\alpha}=\delta_{i j} E^{\alpha}$ (the $\delta_{i j}$ 's being Kronecker symbols). Since $F$ is a 
real number, denoting by $\alpha^{*}$ the transpose of a matrix $a$, one has

$$
\begin{aligned}
\sum_{i, j} Q_{i j}^{\alpha} & \int_{0}^{t} x(s)^{i} x(s)^{j} d s=E^{\alpha} \int_{0}^{t} x(s) x(s)^{*} d s \\
= & \frac{t}{2}\left(\left(3 M M^{*}+x_{0} x_{0}^{*}\right)\left(F^{2}\right)^{-1}-\left(x_{0} M^{*}+M x_{0}^{*}\right) F^{-1}+x_{0} x_{0}^{*}\right) \\
& -\left(M x_{0}^{*}+x_{0} M^{*}\right) F^{-2} \cos (t \sqrt{F}) \\
& +\left(M x_{0}^{*}+x_{0} M^{*}-2 M M^{*} F^{-1}\right) F^{-3 / 2} \sin (t \sqrt{F}) \\
& --\left(2 F^{-1}\left(M \dot{x}_{0}^{*}+\dot{x}_{0} M^{*}\right)-\left(\dot{x}_{0} x_{0}^{*}+x_{0} \dot{x}_{0}^{*}\right)\right) F^{-1} \sin ^{2}(t \sqrt{F}) \\
& +\left(\left(M M^{*}-\dot{x}_{0} \dot{x}_{0}^{*}\right) F^{-1}-M x_{0}^{*}-x_{0} M^{*}\right)\left(4 F^{3 / 2}\right)^{-1} \sin (t \sqrt{F}) .
\end{aligned}
$$

For $H_{R}(p, q, 1)$ one can also describe $z(t)$ in elementary functions using (6):

$$
z(t)=z_{0}+t E+x(t) y(t)-x_{0} y_{0}-\int_{0}^{t} \dot{x}(s) y(s) d s
$$

and since $\dot{x} y=C y-E y^{*} y$ along each geodesic, the integral in (13) can be expressed in elementary functions, as in (12), by virtue of (6) of $\S 1$. Notice that in $H_{R}(p, q, r)$ we must use double indices $i_{u}, v_{\alpha}, j_{\beta}$ on $X, Y, Z$, associated to the usual matric basis $(i, j \in\{1, \ldots, p\}, u, v \in\{1, \ldots, q\}, \alpha, \beta \in\{1, \ldots, r\})$. The coefficients of the matrix product $B$ with respect to the usual matric bases are

$$
B_{i_{u} v_{\alpha}}^{j_{\beta}}=\delta_{i}^{j} \delta_{u}^{v} \delta_{\alpha}^{\beta}
$$

and $C, E$ are matrices whose components are those in (4) and (6), $C=\left(C^{i_{u}}\right)$, $E=\left(E^{j_{\alpha}}\right)$. When $p$ and $r>1$ it is possible to detail the third term in $\S 1(7)$ as a sum of several power series, although the full expression is too lengthy to be included here. Let us remark that the family of groups $H_{R}(p, q, 1)$ were studied in [1] from a contact geometry viewpoint. In [3] A. Kaplan found the geodesics of the mentioned groups of Heisenberg type considering the corresponding metric §1(3) on them; in this case $z(t)$ can also be expressed in elementary functions.

3. Curvature and Ricci tensor of $\left(G_{B}, g\right)$. Let $\nabla$ be the Levi-Civita connection associated to the metric tensor $g$ in $\S 1(3)$. Denote with capital Latin indices $I, J, K, \ldots$ any of the indices in the finite families $\{i\},\{u\},\{\alpha\}$. A left-invariant frame, dual of $\S 1(2)$ is $\left\{U_{I}\right\}$,

$$
U_{i}=X_{i}, \quad U_{u}=Y_{u}, \quad U_{\alpha}=Z_{\alpha},
$$

where $\left\{X_{i}, Y_{u}, Z_{\alpha} ; i \in \Xi, u \in \Upsilon, \alpha \in \Theta\right\}$ has been defined in $\S 1(4)$. The connection coefficients $\gamma_{J K}^{I}$ of $\nabla$ with respect to $\left\{U_{I}\right\}$ are given by

$$
\nabla_{U_{J}} U_{I}=\sum_{K} \gamma_{I J}^{K} U_{K}
$$

Since the only nontrivial structure coefficients of the Lie algebra of $G_{B}$ are

$$
c_{i u}^{\alpha}=\varsigma^{\alpha}\left(\left[X_{i}, Y_{u}\right]\right)=B_{i u}^{\alpha}=-c_{u i}^{\alpha},
$$

it follows that at most the only nonzero connection coefficients (up to the antisymmetry condition $\gamma_{J K}^{I}+\gamma_{I K}^{J}=0$ ), are the constants

$$
\gamma_{u \alpha}^{i}=B_{i u}^{\alpha} / 2, \quad \gamma_{\alpha u}^{i}=B_{i u}^{\alpha} / 2, \quad \gamma_{u i}^{\alpha}=B_{i u}^{\alpha} / 2, \quad i \in \Xi, u \in \Upsilon, \alpha \in \Theta .
$$


Therefore the components of the curvature tensor, $R(\cdot, \cdot)=[\nabla, \nabla]-\nabla_{[\cdot, \cdot]}$, with respect to the frame $\left\{U_{A}\right\}$ given in (1), are constant,

$$
R_{J K L}^{I}=\sum_{H}\left(\gamma_{J L}^{H} \gamma_{H K}^{I}-\gamma_{J K}^{H} \gamma_{H L}^{I}-\gamma_{J H}^{I} c_{K L}^{H}\right),
$$

and bearing in mind (3), (4) and (5) one finds that the only non-necessarily zero components of the Riemann-Christoffel tensor, $R_{I J K L}=\sum_{H} g_{I H} R_{J K L}^{H}$ (up to permutations of indices not changing its modulus), are

$$
\begin{gathered}
R_{i \alpha j \beta}=\frac{1}{4} \sum_{u} B_{j u}^{\alpha} B_{i u}^{\beta} \\
R_{u \alpha v \beta}=\frac{1}{4} \sum_{i} B_{i v}^{\alpha} B_{i u}^{\beta} \\
R_{i u j v}=-\frac{1}{4} \sum_{\alpha}\left(B_{j u}^{\alpha} B_{i v}^{\alpha}+2 B_{i u}^{\alpha} B_{j v}^{\alpha}\right) \\
R_{\alpha \beta u v}=\frac{1}{4} \sum_{i}\left(B_{i v}^{\alpha} B_{i u}^{\beta}-B_{i u}^{\alpha} B_{i v}^{\beta}\right) \\
R_{i j u v}=\frac{1}{4} \sum_{\alpha}\left(B_{i v}^{\alpha} B_{j u}^{\alpha}-B_{i u}^{\alpha} B_{j v}^{\alpha}\right) \\
R_{i j \alpha \beta}=\frac{1}{4} \sum_{u}\left(B_{j u}^{\alpha} B_{i u}^{\beta}-B_{i u}^{\alpha} B_{j u}^{\beta}\right)
\end{gathered}
$$

where $i, j \in \Xi, u, v \in \Upsilon, \alpha, \beta \in \Theta$. Hence, the Ricci tensor, $\rho_{I J}=\sum_{H} R_{I H J}^{H}$, does not depend on the components (10), (11) and (12). At most its only nontrivial coefficients in the moving frame (1) are

$$
\begin{aligned}
\rho_{\alpha \beta} & =\frac{1}{2} \sum_{i, u} B_{i u}^{\alpha} B_{i u}^{\beta}, \\
\rho_{u v} & =-\frac{1}{2} \sum_{i, \alpha} B_{i u}^{\alpha} B_{i v}^{\alpha}, \\
\rho_{i j} & =\frac{1}{2} \sum_{u, \alpha} B_{i u}^{\alpha} B_{j u}^{\alpha} .
\end{aligned}
$$

As a consequence, no connected and simply connected Lie HR-group $G_{B}$ endowed with the left invariant metric tensor $g$, $\S 1(3)$, can be Einstein, i.e.: $g_{I J}=K \rho_{I J}$ is not possible, since at least one component $\rho_{u u}$ is strictly negative ( $B$ is not the zero map), whereas $g_{I J}=\delta_{I J}$. The same is true for all their nilmanifolds $\Gamma \backslash G_{B}$ with the metric induced by $g$, because they are locally isometric to $\left(\tilde{G}_{B}, g\right)$. The scalar curvature $\sigma, \sigma=\sum_{J} \rho_{J J}$, is therefore

$$
\sigma=-\frac{1}{2} \sum_{i, u, \alpha}\left(B_{i u}^{\alpha}\right)^{2}<0 .
$$

On an arbitrary compact HR-nilmanifold $\Gamma \backslash G_{B}$, every differentiable vector field conformal for the metric $\bar{g}$ induced by $g, \S 1(3)$, must in fact be an infinitesimal isometry. This is a consequence of (16) and a well-known theorem of Lichnerowicz 
[5, p. 134]. Notice that Theorem 2 in this paper improves the preceding statement, for HR-spaces as well as Proposition 2 in [6].

Let us recall that on every Riemannian manifold $(M, g)$ there exists a $(1,3)$ tensor field $C$ which is invariant under conformal transformations, called Weyl conformal tensor or conformal curvature tensor. Its components with respect to an arbitrary moving frame $\left\{U_{J}\right\}$ are

$$
\begin{aligned}
C_{J K L}^{I}= & R_{J K L}^{I}+\left(\sigma\left(\delta_{K}^{I} g_{J L}-\delta_{L}^{I} g_{J K}\right) /(n-1)(n-2)\right) \\
& -\left(\left(\delta_{K}^{I} \rho_{J L}-\delta_{L}^{I} \rho_{J K}\right)+\sum_{H} g^{I H}\left(g_{J K} \rho_{H L}-g_{J L} \rho_{H K}\right)\right) /(n-2),
\end{aligned}
$$

where $g^{I J}$ are the components of the metric tensor induced by $g$ on covectors, and where $n$ is the dimension of $M$. When $n>3(M, g)$ is locally conformally Euclidean, (i.e.: conformally flat), if and only if the conformal curvature is zero. For $n=3$ this tensor is always zero, and to be conformally flat is then characterized by the vanishing of the Schouten tensor

$$
C_{I J K}=\rho_{I J, K}-\rho_{I K, J}-\left(g_{I J} \sigma_{, K}-g_{I K} \sigma_{, J}\right) / 4,
$$

a comma preceding an index $K$, for a given component of a $(r, s)$-tensor field $A$ having coefficients $A_{J_{1} \ldots J_{s}}^{I_{1} \ldots I_{r}}$ with respect to a moving frame $\left\{U_{J}\right\}$, meaning the component in the same indices of the tensor $\nabla_{U_{K}} A$, that is to say,

$$
A_{J_{1} \ldots J_{s}, K}^{I_{1} \ldots I_{r}}=\left(\nabla_{U_{K}} A\right)_{J_{1} \ldots J_{s}}^{I_{1} \ldots I_{r}}
$$

4. Proof of Theorem 2. Let us denote $p=\operatorname{dimension}$ of $X=\operatorname{cardinal}(\Xi)$, $q=\operatorname{dim}(Y)=\operatorname{card}(\Upsilon), r=\operatorname{dim}(Z)=\operatorname{card}(\Theta), n=p+q+r=\operatorname{dim}\left(G_{B}\right)$, $(p, q, r \geq 1)$, and call

$$
|B|^{2}=\sum_{j, v, \beta}\left(B_{j v}^{\beta}\right)^{2} .
$$

Since the map $B$ is not zero it follows that $|B|^{2}>0$. Analogously, let us denote

$$
\left|B_{i}\right|^{2}=\sum_{v, \beta}\left(B_{i v}^{\beta}\right)^{2}, \quad\left|B_{u}\right|^{2}=\sum_{j, \beta}\left(B_{j u}^{\beta}\right)^{2}, \quad\left|B^{\alpha}\right|^{2}=\sum_{j, v}\left(B_{j v}^{\alpha}\right)^{2}
$$

$(i, j \in \Xi ; u, v \in \Upsilon ; \alpha, \beta \in \Theta)$. Notice that

$$
|B|^{2}=\sum_{i}\left|B_{i}\right|^{2}=\sum_{u}\left|B_{u}\right|^{2}=\sum_{\alpha}\left|B^{\alpha}\right|^{2}>0 .
$$

Let us suppose that all components $C_{J K L}^{I}$ of the conformal curvature tensor, $\S 3(17)$, are zero on the universal covering $\tilde{G}_{B}$ of $G_{B}$, endowed with the metric tensor $g, \S 1(3)$. We shall arrive at the contradiction $B=0$. Therefore $\left(\tilde{G}_{B}, g\right)$ cannot be conformally flat. The same will hold for $M=\Gamma \backslash G_{B}$ with the induced metric $\bar{g}$, because it is locally isometric to $\left(\tilde{G}_{B}, g\right)$. In fact, by hypothesis there exists at least one coefficient $B_{i u}^{\alpha}$ different from zero. Fixing its indices, it follows from (3), (7), (8), (9) of $\S 3$ that

$$
R_{\alpha i \alpha}^{i}>0, \quad R_{\alpha u \alpha}^{u}>0, \quad R_{u i u}^{i}>0
$$


and therefore, using (7), (13), (15), (16) of $\S 3$ and the fact that $g_{I J}=\delta_{I J}$ in the frame $\S 1(4)$, we obtain

$$
C_{\alpha i \alpha}^{i}=\frac{1}{4} \sum_{v}\left(B_{i v}^{\alpha}\right)^{2}-\frac{|B|^{2}(n-1)^{-1}-\left|B^{\alpha}\right|^{2}+\left|B_{i}\right|^{2}}{2(n-2)} .
$$

Let us fix the index $i$ in (5), sum in all indices $\alpha \in \Theta$, and then multiply the result by $2(2-n)$. We get

$$
2(2-n) \sum_{\alpha} C_{\alpha i \alpha}^{i}=\left(1+\frac{r}{h-1}\right)|B|^{2}-\left(r+\frac{n-2}{2}\right)\left|B_{i}\right|^{2} .
$$

Summing now in those indices $i \in \Xi$, it follows

$$
2(2-n) \sum_{i, \alpha} C_{\alpha i \alpha}^{i}=\left(p\left(1+\frac{r}{n-1}\right)-\left(r-1+\frac{n}{2}\right)\right)|B|^{2} .
$$

By (3), if the conformal curvature tensor is zero we arrive at

$$
p-r+1-n / 2+p r /(n-1)=0 .
$$

In the same way, starting from $C_{\alpha u \alpha}^{u}$ and $C_{u i u}^{i}$, one finds in each case that if $\left(\tilde{G}_{B}, g\right)$ is conformally flat then

$$
\begin{array}{r}
q-r+1-n / 2+q r /(n-1)=0, \\
p+q+3-3 n / 2-p q /(n-1)=0,
\end{array}
$$

Subtracting (9) from (8) we find

$$
(p-q)(1+r /(n-1))=0 .
$$

Since $1+r /(n-1)>0$, the assumption " $\left(\tilde{G}_{B}, g\right)$ is conformally flat" yields a contradiction when $p$ and $q$ are not equal. So let us suppose $p=q$. Under this hypothesis, (8) and (10) give respectively

$$
\begin{gathered}
3 r^{2}+4 p r-4 p-5 r+2=0, \\
6 p^{2}+8 p r-14 p+3 r^{2}-9 r+6=0 .
\end{gathered}
$$

This system has no other positive integer solution than $p=r=1$. Hence $n=3$. But in this case $X=Y=Z=R$, and there is only one index of type " $i$ ", " $u$ ", " $\alpha$ ", respectively. Keeping in mind (4), (13), (14) and (15) of $\S 3$ one deduces that the Schouten tensor $§ 3(18)$ has some nontrivial components, for example

$$
C_{i u \alpha}=\rho_{i u, \alpha}-\rho_{i \alpha, u}=-B^{3} / 2,
$$

where $B$ is the nonzero real number such that $B(x, y)=B x y$. Thus, in all cases conformal flatness does not occur.

Since $g$ is left-invariant, $(M, \bar{g})$ is a nonconformally flat, locally homogeneous Riemannian space. A theorem of Kulkarni, for dimension $n>3$ [4, Theorem 12.2], and Yau, when dimension $>2$ [12, Proposition 4], says that under these conditions every conformal transformation must be an isometry. Points 2 and 3 are proved. To see that $M$ is not projectively flat is equivalent to proving that its universal 
covering is not of constant curvature. This immediately follows by choosing a nonzero coefficient $B_{i u}^{\alpha}$ of $B$, which yields, by virtue of $\S 3(9),(8)$

$$
R_{i u i u}=-\frac{3}{4} \sum_{\beta}\left(B_{i u}^{\beta}\right)^{2}<0 ; \quad R_{i \alpha i \alpha}=\frac{1}{4} \sum_{v}\left(B_{i v}^{\alpha}\right)^{2}>0 .
$$

5. Final remarks. Straightforward computations show that if a connected and simply connected Lie HR-group $G_{B}$ endowed with the metric tensor $g$ has diagonal Ricci tensor with respect to the orthonormal moving frame $\S 1(4)$ then the Ricci tensor is not parallel, and thus the space and their nilmanifolds are not locally symmetric. This situation appears for the groups $H_{\mathbf{R}}(p, q, r)$ mentioned in $\S 2$. One can also prove under that hypothesis, reasoning as in [6, Proposition 2], that no nilmanifold of $G_{B}$ is a 5 -space in the sense of Sasaki-Yano, and thus Theorem 2 cannot be obtained from [11, Theorem 2] in that case.

Let us recall that for a nilpotent Lie group to admit discrete uniform subgroups is equivalent to the existence of rational structure coefficients with respect to at least one basis of its Lie algebra [8]. For a connected and simply connected Lie HR-group $G_{B}$ this fact is characterized by the existence of bases $\left\{e_{i} ; i \in \Xi\right\}$ of $X,\left\{f_{u} ; u \in \Upsilon\right\}$ of $Y$ and $\left\{g_{\alpha} ; \alpha \in \Theta\right\}$ of $Z$ such that the coefficients $B_{i u}^{\alpha}$ of $B$ be integer numbers, because of $\S 3(3)$. In these conditions, for each family $K$ of positive integers,

$$
K=\left\{k^{i}, k^{u} \in \mathbf{Z} ; i \in \Xi, u \in \Upsilon, k^{i}>0, k^{u}>0\right\},
$$

we can define the discrete subgroup $\Gamma_{K}$ of $G_{B}$ given by

$$
\begin{aligned}
\Gamma_{K}=\left\{(x, y, z) \in G_{B} ; x=\sum_{i} k^{i} x^{i} e_{i}, y=\sum_{u} k^{u} y^{u} f_{u}\right. & \\
& \left.z=\sum_{\alpha} z^{\alpha} g_{\alpha}, x^{i}, y^{u}, z^{\alpha} \in Z\right\}
\end{aligned}
$$

which is uniform, since it defines a fundamental domain $D$ in $G_{B}$ with compact closure projecting onto $\Gamma_{K} \backslash G_{B}$,

$$
D=\left\{(a, b, c) \in G_{B} ; 0 \leq a^{i}<k^{i}, 0 \leq b^{u}<k^{u}, 0 \leq c^{\alpha}<1\right\} .
$$

Therefore the volume of $M=\Gamma_{K} \backslash G_{B}$ is

$$
\operatorname{vol}(M)=\int_{M} \bar{\omega}=\int_{D} \omega=\left(\prod_{i \in \Xi} k^{i}\right)\left(\prod_{u \in \Upsilon} k^{u}\right)
$$

where $\bar{\omega}$ and $\omega$ are the canonical volume forms on $(M, \bar{g})$ and $\left(G_{B}, g\right)$ respectively, $g$ the Riemannian metric $\S 1(3)$. One gets nonisometric HR-nilmanifolds via (4).

We would like to thank T. Hangan and R. Lutz for their guidance and help during a period of two years at the University of Haute Alsace in Mulhouse, France.

\section{REFERENCES}

1. M. Goze and Y. Haraguchi, Sur les r-systèmes de contact, C. R. Acad. Sci. Paris 294 (1982), 95-97.

2. G. Jensen, The scalar curvature of left-invariant Riemanmnian metrics, Indiana Univ. Math. J. 20 (1971), 1125-1144. 
3. A. Kaplan, On the geometry of groups of Heisenberg type, Bull. London Math. Soc. 15 (1983), 35-42.

4. R. Kulkarni, Curvature structures and conformal transformations, J. Differential Geom. 4 (1970), 425-451.

5. A. Lichnerowicz, Géométrie des groupes de transformations, Dunod, Paris, 1958.

6. J. F. T. Lopera, Conformal vector fields on a certain class of nilpotent groups, Research Notes in Math., no. 131, Pitman, London, 1985, pp. 76-84.

7. __ Sur les propriétés géométriques de certaines familles d'espaces homogènes, Publ. I.R.M.A., Univ. de Strasbourg, 1985, pp. 22-38.

8. A. I. Mal'cev, On a class of homogeneous spaces, Amer. Math. Soc. Transl. 39 (1951), 266-307.

9. T. Milnor, Curvatures of left-invariant metrics on Lie groups, Adv. in Math. 21 (1976), 293-329.

10. H. Reiter, Über den Satz von Wiener und lokalkompakte Gruppen, Comment. Math. Helv. 49 (1974), 333-364.

11. N. Tanaka, Conformal connections and conformal transformations, Trans. Amer. Math. Soc. 92 (1959), 168-190.

12. S. T. Yau, Remarks on conformal transformations, J. Differential Geom. 8 (1973), 369-381.

Departamento de Geometria y Topología Facultad de Matemáticas, SanTIAGO DE COMPOSTELA, SPAiN 